\title{
Infinitely Divisible Distribution Functions of Class $L$ and the Lee-Yang Theorem
}

\author{
Joël De Coninck \\ Université de l'Etat, Faculté des Sciences, B-7000-Mons, Belgium
}

\begin{abstract}
It is shown that the free-energy density of a large class of ferromagnets satisfying the Lee-Yang property is to be connected with the limit characteristic function of a suitably renormalized sum of independent and non-identically distributed random variables. Using the canonical representation formulae of such characteristic functions, various chains of inequalities are derived for the Ursell functions.
\end{abstract}

\section{Introduction}

In spite of considerable efforts, only a few models have been exactly solved in statistical mechanics. It is therefore of great interest to find general properties of the free-energy density or other related variables at the thermodynamic limit. An important contribution along this direction was achieved many years ago by Lee and Yang $[1,2]$. If $Z_{N}(\beta, \beta h)$ denotes the partition function of a $d$-dimensional Ising ferromagnet with pairwise interactions (as usual, $\beta$ is the reciprocal temperature, $h$ is the external field and $N$ is the number of spins), they proved that the zeros of $Z_{N}(\beta, \beta h)$ as a function of $z=\exp (2 \beta h)$ all lie on the unit circle in the complex $z$ plane.

A number of interesting results have been derived since using various forms of the Lee-Yang theorem (e.g. [3-5]). Recently, J. De Coninck and Ph. de Gottal [6] established a connection between the Lee-Yang theorem and the set of infinitely divisible distribution functions (see below). This leads to various inequalities for the Ursell functions of finite systems.

In this paper, we extend this analysis by showing that such a connection also holds when the thermodynamic limit is considered. Before presenting our results, let us recall some terminology $([8,9])$.

Let $\left(X_{i j}\right)_{l \geq 1,1 \leqq j \leqq k_{i}}$ be a double sequence of random variables that are mutually independent for a fixed $i$ and subject to the condition of infinite smallness, i.e. for every $\varepsilon>0$.

$$
\max _{1 \leqq k \leqq k_{n}} P\left\{\left|X_{n k}\right| \geqq \varepsilon\right\} \rightarrow 0 \quad \text { as } n \rightarrow \infty .
$$


The set of distributions that are limits of the distributions of sums $\sum_{k=1}^{k_{n}} X_{n k}$ of such random variables is the set of infinitely divisible distribution functions.

If $\left(X_{k}\right)_{k \geqq 1}$ is a sequence of independent random variables and $\left(a_{n}\right)_{n \geqq 1}$ is a sequence of positive constants such that the $X_{n k}=X_{k} / a_{n}$ are infinitely small for $k=1,2, \ldots, n$, the set of distribution functions which are limits of distributions of sums

$$
\left(1 / a_{n}\right) \sum_{k=1}^{n} X_{k}-b_{n}
$$

where $\left(b_{n}\right)_{n \geqq 1}$ is a sequence of real numbers, is the class $L$. In order that the limit of (2) exists, the sequences $\left(a_{n}\right)$ and $\left(b_{n}\right)$ must be suitably chosen.

Under the additional restriction that the random variables $X_{1}, X_{2} \ldots$ have identical distributions, the limit distributions of sums of the form (2) constitutes the set of stable distributions.

We first consider the class of $d$-dimensional ferromagnets with pairwise interactions, symmetric a priori free spin distribution with bounded support and satisfying the Lee-Yang theorem. If the free-energy density exists at the thermodynamic limit and is continuous with respect to the external field, we establish a connection between this free-energy density and the logarithm of the characteristic function of an infinitely divisible distribution (Sect. 2). Using the canonical representation formulae of these characteristic functions, we derive various chains of inequalities (including new ones) for the Ursell functions of the magnetisation variable. In particular it is shown that the critical gap exponent ([10]) inequalities $\Delta_{4} \leqq \Delta_{6} \leqq \ldots$ are direct consequences of such representation formulae (Sect. 3).

Furthermore, we also prove that the free-energy density of these models is to be connected with the characteristic function of a probability distribution which belongs to the class L. Using the GKS ([7]) inequality, we also show that this characteristic function cannot be stable. This means that, to each $d$-dimensional ferromagnet we consider, we may associate the limit characteristic function of a suitably renormalized sum of independent and non-identically distributed random variables. This leads to some new inequalities for the zero-field Ursell functions above the critical temperature (Sect. 4).

For $d$-dimensional Ising ferromagnets, we derive more restrictions on the subclass of self-decomposable characteristic functions associated to the free energy (Sect. 5).

We finally present in Sect. 6 some conclusions and perspectives.

\section{Infinitely Divisible Distributions}

Let us consider a lattice $\mathbb{Z}^{d}$ at each vertex $j=\left(j_{1}, \ldots, j_{d}\right)$ of which we associate a spin variable $S_{j}$ taking the values $\sigma_{j}$ with the a priori free spin probability distribution $\rho$. The Van Hove sequence of blocks $\left(\Lambda_{n}\right)_{n \geqq 1}$ will be defined by

$$
\Lambda_{n}=\left\{j \in \mathbb{Z}^{d}: 0 \leqq\left|j_{k}\right|<n \quad \text { for } k=1, \ldots, d\right\} .
$$

The energy of a given block configuration will be taken as (free boundary conditions)

$$
E_{\Lambda_{n}}\left(\sigma_{1}, \ldots, \sigma_{\left|\Lambda_{n}\right|}\right)=-\sum_{j, k \in \Lambda_{n}} J_{k j} \sigma_{k} \sigma_{j}-h \sum_{k \in \Lambda_{n}} \sigma_{k}
$$


where $J_{i j} \geqq 0$ is such that the thermodynamic limit exists (see for instance ref [7]). The joint probability distribution function of a fixed configuration is given by the finite Gibbs state:

$$
d \mu_{\Lambda_{n}}\left(\sigma_{1}, \ldots, \sigma_{\mid \Lambda_{n}}\right)=Z_{\Lambda_{n}}^{-1}(\beta, \beta h) \exp \left\{-\beta E_{\Lambda_{n}}\left(\sigma_{1}, \ldots, \sigma_{\mid \Lambda_{n}}\right)\right\} \prod_{k \in \Lambda_{n}} d \rho\left(\sigma_{k}\right),
$$

where $Z_{\Lambda_{n}}(\beta, \beta h)$ is the partition function normalizing the Gibbs state. The random variable magnetisation $M_{n}$ is defined by

$$
M_{n}=\sum_{k \in \Lambda_{n}} S_{k} .
$$

Let us consider the moment generating function of this random variable with respect to the corresponding finite Gibbs state at zero external field. It is easily shown that for any real $t$,

$$
\left\langle\exp \left(t M_{n}\right)\right\rangle=Z_{\Lambda_{n}}(\beta, t) / Z_{\Lambda_{n}}(\beta, 0) .
$$

In the following, we shall restrict ourselves to symmetric free spin probability distribution with bounded support (i.e. $\exists a, b \in \mathbb{R}: \forall \varepsilon>0: \quad \rho(a-\varepsilon)=0$ and $\rho(b+\varepsilon)=1)$ satisfying the Lee-Yang theorem $([4,5])$. This ensures that the zeros of $\left\langle\exp \left(t M_{n}\right)\right\rangle$ are purely imaginary numbers. Using elementary properties of probability distributions, the following result has been proven in [6].

Lemma 1. For the class of d-dimensional ferromagnets with pairwise interactions, if the free spin distribution is symmetric, has a bounded support and verifies the LeeYang property, then there exists an infinitely divisible characteristic function $\phi_{n}$ such that for any real $t$.

$$
\left\langle\exp \left(t M_{n}\right)\right\rangle=1 / \phi_{n}(t)
$$

Remarks.

1) ([8]) A characteristic function $f(t)$ is said to be infinitely divisible if, and only if, for every positive integer $q$ there exists a characteristic function $f_{q}(t)$ such that $f(t)=\left[f_{q}(t)\right]^{q}$.

2) ([6]) Formula (7) also holds for random variables of Newman's type $\mathscr{L}$ ([14]), in particular for the $\phi^{4}$ lattice field theory.

At the thermodynamic limit, one is interested in the following quantity:

$$
w(t)=\lim _{\Lambda_{n} \uparrow \mathbb{Z}^{d}}\left\langle\exp \left(t M_{n}\right)\right\rangle^{1 / \Lambda_{n} \mid} .
$$

Using (7), one gets

$$
\begin{aligned}
w(t) & =\lim _{\Lambda_{n} \uparrow \mathbb{Z}^{a}} \exp \left\{\frac{1}{\left|\Lambda_{n}\right|} Z_{\Lambda_{n}}(\beta, t)-\frac{1}{\left|\Lambda_{n}\right|} \ln Z_{\Lambda_{n}}(\beta, 0)\right\} \\
& =\exp \{-\beta g(\beta, t)+\beta g(\beta, 0)\},
\end{aligned}
$$

where $g$ denotes the free-energy density at the thermodynamic limit, i.e.

$$
\beta g(\beta, t)=-\lim _{\Lambda_{n} \uparrow \mathbb{Z}^{d}} \frac{1}{\left|\Lambda_{n}\right|} \ln Z_{\Lambda_{n}}(\beta, t) .
$$


(We implicity assume here that the interaction $J_{i j}$ is such that (11) indeed exists.) On the other hand, using Lemma 1, one has that

$$
w(t)=\lim _{\Lambda n \uparrow \mathbb{Z}^{d}} 1 /\left[\phi_{n}(t)\right]^{1 /\left|\Lambda_{n}\right|} .
$$

Since $\phi_{n}(t)$ is infinitely divisible, one knows that $\left[\phi_{n}(t)\right]^{1 /\left|\Lambda_{n}\right|}$ is also infinitely divisible for any $n([9])$. Using the continuity theorem on the convergence of a sequence of characteristic functions, one has that, if the free energy density exists at the thermodynamic limit and is a continuous function of the external field, then there exists an infinitely divisible characteristic function $\phi$ such that for any real $t$

$$
\exp \{-\beta g(\beta, t)+\beta g(\beta, 0)\}=1 / \phi(t) \text {. }
$$

Indeed, if a sequence of infinitely divisible characteristic functions converges to some characteristic function, then this last one is also infinitely divisible ([8]). This allows us to formulate the following.

Theorem 1. For d-dimensional ferromagnets with pairwise interactions and a free spin distribution $\rho$ satisfying the hypotheses of Lemma 1, if, at the thermodynamic limit, the free-energy density exists and is a continuous function of the external field then there exists a bounded non-decreasing function $G(x)$ such that:

$$
\beta g(\beta, t)-\beta g(\beta, 0)=\int_{-\infty}^{+\infty}[\cos (t x)-1] \frac{1+x^{2}}{x^{2}} d G(x) .
$$

The function under the integral sign is defined to be equal to $-t^{2} / 2$ for $x=0$.

Proof. Straightforward using (14), the Lévy-Khintchine canonical representation (e.g. [8]) and the fact that $g(\beta, t)=g(\beta,-t)$.

Let us now introduce the Ursell functions for infinite systems. As usual, they are defined by

$$
u_{n}(\beta h)=\left[\partial_{\beta h}^{n}(-\beta g)\right](\beta h)
$$

for a fixed temperature $\beta^{-1}$. One of the main interests of the representation (15) is that the external field dependence of the right-hand side is very simple (the temperature dependence is to be found in the $G$ function). Moreover, if the variance of the random variable associated to $\phi(t)$ in $(14)$ is finite, i.e. $u_{2}(0)<+\infty$, then we may use the Kolmogorov canonical representation ([8]) which leads to the following:

Theorem 2. For d-dimensional ferromagnets with pairwise interactions and a free spin distribution $\rho$ satisfying the hypotheses of Lemma 1, if, at the thermodynamic limit,

a) the free-energy density exists and is a continuous function of the external field, b) $u_{2}(0)<+\infty$ for some temperature $\beta^{-1}$,

then, there exists a non-decreasing and bounded function $K(x)$ such that, for this temperature $\beta^{-1}$,

$$
\beta g(\beta, t)-\beta g(\beta, 0)=\int_{-\infty}^{+\infty}[\cos (t x)-1] d K(x) / x^{2},
$$


where the function under the integral sign is defined to be equal to $-t^{2} / 2$ for $x=0$ and where $K(+\infty)=u_{2}(0)$.

These theorems establish the connection between the free-energy density at the thermodynamic limit and the logarithm of the characteristic function of an infinitely divisible distribution function. In the following, we show how non-trivial inequalities for the Ursell functions at zero external field follow as direct consequences of the representation formula (17). To that effect, one obviously has to assume that the Ursell functions exist and therefore, if there is a critical temperature $T_{c}=\beta_{c}^{-1}$ defined by

$$
\beta_{c}=\sup \left\{\beta: \lim _{t>0} u_{1}(t)=0\right\},
$$

one has to distinguish between the two regimes $\beta>\beta_{c}$ and $\beta<\beta_{c}$. This will be developed in the following paragraph.

\section{General Inequalities}

a) Below the critical temperature. Since for $\beta>\beta_{c}$,

$$
\lim _{t \gg 0} u_{1}(t) \neq \lim _{t \geq 0} u_{1}(t)
$$

the characteristic function $\phi(t)$ is not differentiable at the origin so that the corresponding moments, and in particular $u_{2}(0)$, do not exist. Below the critical temperature, one has therefore to use the representation formula (15). A typical example of such behavior is given by the well known Cauchy distribution which is expected to hold as $\beta \rightarrow \infty$ at least for $d$-dimensional Ising ferromagnets (see also below):

$$
\phi(t)=\exp (-|t|)
$$

where the Lévy-Khintchine spectral function is given by

$$
G(x)=(1 / \pi) \operatorname{arctg} x+1 / 2 .
$$

b) Above the critical temperature. Above $T_{c}$, it is expected that $u_{2}(0)<+\infty$, though it does not follow from our definition (18). Nevertheless, if we assume that $u_{2}(0)$ will be finite for any $\beta<\beta_{c}$ and, moreover, that the other cumulants $u_{2 n}(0)$ also exist for $n=2, \ldots ;$ then

$$
u_{2 n}(0)=(-1)^{n-1} \int_{-\infty}^{+\infty} x^{2 n-2} d K(x)
$$

By considering

$$
H(x)=K(x) / u_{2}(0)
$$

and using essentially Schwarz's inequality, one gets the following.

Proposition 1. For d-dimensional ferromagnets with pairwise interactions and a free spin distribution $\rho$ satisfying the hypotheses of Lemma 1, if, at the thermodynamic limit, 
a) the free energy density exists and is a continuous function of the external field, b) the Ursell functions $u_{2 n}(0)$ exist for $n=2,3, \ldots$ and for any $\beta<\beta_{c}$,

then they will satisfy the following chains of inequalities

$$
\begin{aligned}
(-1)^{n-1} u_{2 n}(0) & \geqq 0, \\
\frac{\left|u_{4}(0)\right|}{u_{2}(0)} & \leqq\left(\frac{u_{6}(0)}{u_{2}(0)}\right)^{1 / 2} \leqq\left(\frac{\left|u_{8}(0)\right|}{u_{2}(0)}\right)^{1 / 3} \leqq\left(\frac{u_{10}(0)}{u_{2}(0)}\right)^{1 / 4} \ldots, \\
\left|u_{2 n}(t)\right| & \leqq\left|u_{2 n}(0)\right|, \\
2\left|u_{4 n}(0)\right| & \leqq u_{4 n+2}(0)+u_{4 n-2}(0), \\
u_{n}^{2}(t) & \leqq\left|u_{2 n_{1}}(0) \cdot u_{2 n_{2}}(0)\right|, \quad \text { where } n_{1}+n_{2}=n .
\end{aligned}
$$

Proof. Equations (24) and (26) are straightforward using (22), (16) and (17). Equations (27) and (28) may be derived by use of Schwarz's inequality starting from (17). Equation (25) is another way of writing Liapounov's chain of inequalities for $H(x)$ (see for instance [11], p. 229).

Let us now introduce the gap exponents $\Delta_{4}, \Delta_{6} \ldots$ As usual, we shall write $f(x) \sim x^{\omega}$ to mean that

$$
\lim _{x>0} \ln f(x) / \ln x=\omega .
$$

Therefore, if for $\beta<\beta_{c}$ the cumulants behave as ([10])

$$
u_{2 n}(0) \sim\left(T-T_{c}\right)^{-\gamma-2} \sum_{k=2}^{n} \Delta_{2 k}
$$

it follows from (28) that (see also [10])

Corollary 1. With the hypotheses of Proposition 1, if the cumulants $u_{2 n}(0)$ behave as in (30), then

$$
\Delta_{4} \leqq \Delta_{6} \leqq \Delta_{8} \leqq \ldots .
$$

Such inequalities (24-28) and (31), among others (see in particular ref [6]), are direct consequences of the Kolmogorov representation theorem. We have proven so far the validity of Theorems 1 and 2 using essentially the Lee-Yang theorem but the equivalence between these theorems remains an open problem which may well prove fruitful to investigate.

In order to obtain results more precise than the general ones derived until now, one needs a better knowledge of the spectral functions $G(x)$ and $K(x)$.

\section{Class L Distribution Functions}

To study the properties of the spectral functions involved in (15) and (17), let us first consider finite systems $\left(\left|\Lambda_{n}\right|<+\infty\right)$. In that case, one knows that at zero external field

$$
\left\langle\exp \left(t M_{n}\right)\right\rangle^{-1}=\prod_{1}^{\infty}\left(1+t^{2} / t_{j}^{2}\right)^{-1}
$$


for any real $t ;\left(t_{j}\right)_{\jmath \geqq 1}$ is an infinite family of real numbers such that $0<t_{1} \leqq t_{2} \leqq \ldots$ with $\sum^{\infty}\left(1 / t_{j}^{2}\right)<+\infty$. This is a particular case of Proposition 2 of [4] and has been proven in [6]. As before, let $\phi_{n}$ denote the characteristic function corresponding to the left-hand side of (32). Since the factors in an absolutely convergent product may be taken in any order [18], one gets that for any $0<\alpha<1$ and any real $t$ :

$$
\phi_{n}(t)=\phi_{n}(\alpha t) \cdot h_{n}(\alpha, t),
$$

where

$$
h_{n}(\alpha, t)=\prod_{1}^{\infty}\left[\left(t_{j}^{2}+\alpha^{2} t^{2}\right) /\left(t_{j}^{2}+t^{2}\right)\right] .
$$

Since each factor of the right-hand side of (34) is an infinitely divisible characteristic function when $0<\alpha<1$, it follows, using the continuity theorem and Theorems 2 and 3 of [8], p. 26, that $h_{n}(\alpha, t)$ is also an infinitely divisible characteristic function. With the help of the following:

Lemma 2 ([8]). A distribution function $F(x)$ with characteristic function $f(t)$ belongs to the class $L$ if and only if corresponding to every $0<\alpha<1$ there exists $a$ characteristic function $f_{\alpha}(t)$ such that

$$
f(t)=f(\alpha t) \cdot f_{\alpha}(t),
$$

one gets that the distribution function associated to $\phi_{n}$ belongs to the class $L$.

Remark. It is not difficult to prove that (33) holds in particular for the $\phi^{4}$ lattice field theory.

Going over to the thermodynamic limit, one may reproduce the same kind of arguments as those developed in Sect. 2 to obtain

Theorem 3 For d-dimensional ferromagnets with pairwise interactions and a free spin distribution $\rho$ satisfying the hypotheses of Lemma 1, if, at the thermodynamic limit, the free-energy density exists and is a continuous function of the external field, then for any $0<\alpha<1$ and any real t, there exists an infinitely divisible characteristic function $\phi_{\alpha}$ such that (cf. (14))

$$
\phi(t)=\phi(\alpha t) \cdot \phi_{\alpha}(t) .
$$

Equivalently, (36) means that

$$
\phi_{\alpha}(t)=\exp \left\{-\int_{\alpha t}^{t} u_{1}(h) d h\right\}
$$

is an infinitely divisible characteristic function for any $0<\alpha<1$. Using this elementary property, we are led to new inequalities for the Ursell functions above the critical temperature.

Let us indeed assume that the Ursell functions $u_{2 n}(0)(n=1,2, \ldots)$ exist above the critical temperature. It is then straightforward to show that the cumulants $K_{\alpha, 2 n}$ of $\phi_{\alpha}$ may be written as

$$
K_{\alpha, 2 n}=\left(1-\alpha^{2 n}\right)\left|u_{2 n}(0)\right| .
$$


Since $\phi_{\alpha}$ is infinitely divisible, we may use the Kolmogorov representation formula to get

$$
\ln \phi_{\alpha}(t)=\int_{-\infty}^{+\infty}[\cos (t x)-1] d K_{\alpha}(x) / x^{2}
$$

where $K_{\alpha}(x)$ is the Kolmogorov spectral function associated to $\phi_{\alpha}(t)\left(K_{\alpha}(+\infty)=\right.$ $\left.K_{\alpha, 2}\right)$. Introducing the function

$$
H_{\alpha}(x)=K_{\alpha}(x) / K_{\alpha, 2},
$$

one gets

$$
\left|K_{\alpha, 2 n} / K_{\alpha, 2}\right|=\int_{-\infty}^{+\infty} x^{2 n-2} d H_{\alpha}(x) .
$$

Since for any $0<\alpha<1$, (for instance)

$$
\left[\int_{-\infty}^{+\infty} x^{2} d H_{\alpha}(x)\right]^{2} \leqq \int_{-\infty}^{+\infty} x^{4} d H_{\alpha}(x),
$$

one gets

$$
u_{4}^{2}(0) \leqq D_{\alpha} u_{6}(0) \cdot u_{2}(0)
$$

where

$$
D_{\alpha}=\left(1-\alpha^{6}\right)\left(1-\alpha^{2}\right) /\left(1-\alpha^{4}\right)^{2} .
$$

Choosing the minimum value of $C_{\alpha}$, it leads

$$
u_{4}^{2}(0) \leqq(3 / 4) u_{6}(0) \cdot u_{2}(0)
$$

which is a slight improvement of

$$
u_{4}^{2}(0) \leqq u_{6}(0) \cdot u_{2}(0)
$$

obtained in (25). Other inequalities may obviously be derived using the same method.

Among the class $L$ are the stable distributions. Their characteristic functions, when symmetric, may be written in complete generality ([8]) as

$$
\phi_{s}(t)=\exp \left(-c|t|^{\lambda}\right)
$$

where $0<\lambda \leqq 2$ and $c$ is some positive constant. Using the GKS inequality ([7]), we prove in the following that the Cauchy characteristic function $(\lambda=1)$ is the only one which may appear as a solution of (14) for the class of $d$-dimensional ferromagnets we consider.

We may first notice that $u_{2}(h)(h \neq 0)$ is non-negative as follows easily by use of the GKS inequality ([7]). This implies that $u_{1}(h)$ is a non-decreasing function of its argument. Therefore for any real $t_{1}$ and $t_{2}$ such that $t_{1}>t_{2}>0$,

$$
\begin{aligned}
\int_{0}^{t_{1}+t_{2}} u_{1}(h) d h & \geqq \int_{0}^{t_{1}} u_{1}(h) d h+t_{2} u_{1}\left(t_{1}\right) \\
& \geqq \int_{0}^{t_{1}} u_{1}(h) d h+t_{2} u_{1}\left(t_{2}\right) \\
& \geqq \int_{0}^{t_{1}} u_{1}(h) d h+\int_{0}^{t_{2}} u_{1}(h) d h .
\end{aligned}
$$


As a direct consequence of this inequality (48.c), one gets that for any real $t$,

$$
\phi(2 t) \leqq[\phi(t)]^{2} \text {. }
$$

By successive applications of this result, we show that for any integer $n$ and any real $t$ :

$$
\phi(t) \leqq[\phi(t / n)]^{n}
$$

which proves that a stable characteristic function with exponent $\lambda<1$ cannot be a solution of (14). On the other hand, since the free spin distributions we consider have a bounded support, there exists some positive constant $R$ such that $\left|u_{1}(h)\right| \leqq R$ for any real field $h$. This leads therefore to

$$
\exp (-R|t|) \leqq \phi(t)
$$

which achieves the proof since this last inequality excludes the $\lambda>1$ cases.

We have thus proven the following.

Theorem 4. For d-dimensional ferromagnets with pairwise interactions and a free spin distribution $\rho$ satisfying the hypotheses of Lemma 1, if, at the thermodynamic limit, the free energy density exists and is a continuous function of the external field, then the characteristic function

$$
\phi(t)=\exp [\beta g(\beta, t)-\beta g(\beta, 0)]
$$

is the limit characteristic function of a suitably renormalised sum of independent and non-identically distributed random variables (expect possibly for the Cauchy case).

Obviously, a precise determination of the class of characteristic functions $\phi\left(\right.$ or $\left.\phi_{\alpha}\right)$ should in principle lead to a better knowledge of the field governed properties of our $d$ dimensional ferromagnets.

As an intriguing by-product of our analysis, one may reformulate the scaling hypotheses of the free energy density in terms of characteristic functions. In particular, at the critical temperature, if

$$
u_{1}(h) \sim|h|^{1 / \delta} \operatorname{sgn}(h),
$$

where the symbol $\sim$ has been defined previously (cf. (29)) and $\delta$ is greater or equal to $1([20])$, we see that

$$
\phi(t) \sim \exp \left(-c|t|^{1+1 / \delta}\right),
$$

where $c$ is some positive constant. This means that $\phi(t)$ is "nearly" stable in the vicinity of the origin, with characteristic exponent $\lambda=1+1 / \delta$ such that, as it should, the first moment exists but not the second one ([19]).

It would therefore be interesting to find additional restrictions on the class of possible candidates $\phi(t)$. Up to now, we have proven that they must belong to the class $L$ and satisfy the inequality (50). In order to obtain more precise restrictions, we study in the following the cases of $d$-dimensional Ising ferromagnets (with the usual up and down free spin distribution). For these models, we derive some new inequalities for the Ursell functions above the critical temperature which, in our opinion, are interesting by themselves and indicate the existence of additional restrictions on the candidates $\phi$. 


\section{5. $d$-Dimensional Ising Ferromagnets}

For the class of such models, one knows that the hypotheses involved until now are indeed satisfied. Moreover, the GHS ([7]) inequality holds for such cases. The concavity of the magnetisation leads us to the following inequalities:

$$
(1 / 2) t u_{1}(t) \leqq \int_{0}^{t} u_{1}(h) d h \leqq t u_{1}(t)
$$

where $u_{1}(t)=-\phi^{\prime}(t) / \phi(t)$. In terms of characteristic functions, one obtains the following result:

$$
(1 / 2) t \phi^{\prime}(t) \geqq \phi(t) \ln \phi(t) \geqq t \phi^{\prime}(t) .
$$

Since the characteristic function of an infinitely divisible distribution never vanishes, this last double inequality holds for any real $t$. As yet, we do not clearly understand the implications of (56).

Another way to get some more restrictions is obtained by the study of the cumulants of $\phi(t)$. As explained previously, one has therefore to consider the $\beta<\beta_{c}$ regime. Many inequalities have already been obtained by Newman ([4]). However, for $d$-dimensional Ising ferromagnets, it is possible to derive additional results.

Let us indeed consider the characteristic function of the magnetisation variable when $\left|\Lambda_{n}\right|<+\infty$. Using (32), one gets for any real $t$

$$
\left\langle\exp \left(i t M_{n}\right)\right\rangle=\prod_{1}^{\infty}\left(1-t^{2} / t_{j}^{2}\right)
$$

Since the spins take the values plus or minus one, one also has

$$
\left\langle\exp \left(i t M_{n}\right)\right\rangle=\sum_{v} p_{v} \cos (v t)
$$

where the $v$ are the values taken by $M_{n}$ with probability $p_{v}$. It is therefore easily seen that if $t_{j}$ is a zero of (57), it is also the case for $t_{j}+2 \pi, t_{j}+4 \pi \ldots$. Introducing the Ursell functions for finite systems at a fixed temperature $\beta^{-1}$ :

one obtains

$$
U_{2 n}(0)=\left.\partial_{\beta h}^{2 n} \ln Z_{\Lambda_{n}}\right|_{\beta h=0},
$$

Proposition 2. For finite d-dimensional Ising ferromagnets, the Ursell functions at zero external field may be written for any $k=1,2,3, \ldots$

$$
(-1)^{k-1} U_{2 k}(0)=2 \Gamma(2 k) /(2 \pi)^{2 k} \sum_{j=1}^{\left|A_{n}\right|} \zeta(2 k, t j / 2 \pi),
$$

where $0<t_{j} / 2 \pi<1$ and $\zeta(2 k, x)$ is the generalized Zeta function (see for instance ref. [13]).

Proof. This follows from the absolute convergence of the product involved in (32) and from the definition of the generalized Zeta function.

$$
\zeta(2 k, x)=\sum_{n=0}^{\infty}(x+n)^{-2 k}, \quad x>0 \text { and } k=1,2, \ldots
$$

1 We are much indebted to D. B. Abraham for explaining to us this consequence of the GHS inequality 
Corollary 2. First lower bounds for the Ursell functions. For d-dimensional Ising ferromagnets, one has that for any $k=1,2, \ldots$

$$
(-1)^{k-1} U_{2 k}(0) \geqq\left|\Lambda_{n}\right| 2 \Gamma(2 k) / \pi^{2 k}
$$

Proof. If $t_{j}$ is a zero of (57), then by symmetry $2 \pi-t_{j}$ is also a zero. On the other hand, since

$$
\zeta(2 k, x)+\zeta(2 k, 1-x) \geqq 1 / x^{2 k}+1 /(1-x)^{2 k}
$$

for $k=1,2, \ldots$ and $0<x<1$, one obtains (61) using the convexity of the function $1 / x^{2 k}$.

This result also holds at the thermodynamic limit for $\beta<\beta_{c}$. In particular, this implies that the susceptibility $\chi_{T}$ at zero field [17] satisfies the following inequality:

$$
\chi_{T} \geqq 2 \beta / \pi^{2}
$$

Corollary 3. Second lower bounds for the Ursell functions. For d-dimensional Ising ferromagnets with $\beta<\beta_{c}$, one has that for any $k=1,2, \ldots$

$$
\left|u_{2 k}(0)\right| \geqq 2 \Gamma(2 k)\left[u_{2}(0) / 4\right]^{k} .
$$

Proof. This follows easily by use of Chebyshev's inequality [12], which states that

Moreover, since for $0<x<1$

$$
\begin{aligned}
{\left[2 \pi^{2} U_{2}(0) /\left|\Lambda_{n}\right|\right]^{k} } & =\left[1 /\left|\Lambda_{n}\right| \sum_{1}^{\left|\Lambda_{n}\right|} \zeta\left(2, t_{j} / 2 \pi\right)\right]^{k} \\
& \leqq 1 /\left|\Lambda_{n}\right| \sum_{1}^{\left|\Lambda_{n}\right|} \zeta^{k}\left(2, t_{j} / 2 \pi\right) .
\end{aligned}
$$

$$
\zeta(2, x)=\sum_{0}^{\infty}(x+j)^{-2} \leqq 1 / x^{2}+\int_{1+x}^{+\infty}(y-1)^{-2} d y \leqq 2 / x^{2},
$$

one obtains

$$
\left[2 \pi^{2} U_{2}(0) /\left|\Lambda_{n}\right|\right]^{k} \leqq\left[(2 \pi)^{2 k}\left|U_{2 k}(0)\right| /\left(2 \Gamma(2 k)\left|\Lambda_{n}\right|\right)\right] \cdot 2^{k}
$$

As $\Lambda_{n} \uparrow Z^{d}$, one gets (63) using [10].

Using Theorem 2 of ref. [12] p. 37, it is not difficult to extend this result. In particular, one gets

$$
\left|u_{2+4 \ldots+2 n}(0)\right| \geqq C_{n}\left|u_{2}(0) \cdot u_{4}(0) \ldots u_{2 n}(0)\right|,
$$

where

$$
C_{n}=\Gamma\left(n^{2}+n\right) /\left[2^{2 n-1} \Gamma(2) \Gamma(4) \ldots \Gamma(2 n)\right] .
$$

The main difficulty is of course to find how these restrictions may help to select some of the characteristic functions of the class $L$.

As a first attempt to solve this problem, we indicate in the following some idea which may well prove to be fruitful.

Using (60), one has that

$$
\left.\left[2 \Gamma(2 k) /(2 \pi)^{2 k}\right] U_{2 k}(0) /\left|\Lambda_{n}\right|\right)=1 /\left|\Lambda_{n}\right| \sum_{1}^{\left|\Lambda_{n}\right|} \zeta\left(2 k, t_{j} / 2 \pi\right) .
$$


Introducing the function $D_{n}$ defined by

$$
D_{n}(\theta)= \begin{cases}0 & \text { for } 0 \leqq \theta<t_{1} / 2 \pi, \\ 1 /\left|\Lambda_{n}\right| & \text { for } t_{1} / 2 \pi \leqq \theta<t_{2} / 2 \pi, \\ 2 /\left|\Lambda_{n}\right| & \text { for } t_{2} / 2 \pi \leqq \theta<t_{3} / 2 \pi, \\ \vdots & \text { for } \theta \leqq t_{\left|\Lambda_{n}\right|} / 2 \pi,\end{cases}
$$

one gets

$$
\left[2 \Gamma(2 k) /(2 \pi)^{2 k}\right]\left|U_{2 k}(0) /\right| \Lambda_{n}||=\int_{0}^{1} \zeta(2 k, \theta) d D_{n}(\theta) .
$$

Since the $\left(D_{n}\right)_{n \geqq 1}$ constitutes a sequence of non-decreasing functions which are uniformly bounded, one knows by use of Helly's first theorem ([11]) that there exists at least one subsequence which converges weakly to some non-decreasing function $D$. Going over to the thermodynamic limit, one has by use of the corollary 8.13 of ref [19] that

$$
\int_{0}^{1} \zeta(2 k, x) d D(x) \leqq\left[2 \Gamma(2 k) /(2 \pi)^{2 k}\right]\left|u_{2 k}(0)\right|,
$$

provided that, for $\beta<\beta_{c}$, the Ursell functions exist. Moreover, one also gets

$$
\left[2 \Gamma(2 k) /(2 \pi)^{2 k}\right]\left|u_{2 k}(0)\right| \geqq \int_{0}^{1}\left(1 / x^{2 k}\right) d D(x),
$$

which, if the density of zeros $\mathscr{D}(x)$ indeed exists, leads to

$$
\left[2 \Gamma(2 k) /(2 \pi)^{2 k}\right]\left|u_{2 k}(0)\right| \geqq \int_{0}^{1}\left(1 / x^{2 k}\right) \mathscr{D}(x) d x .
$$

That the left-hand side of (70) remains finite for any $k=1,2, \ldots$ obviously suggests the existence of the so-called Lee-Yang gap, i.e.

$$
\exists \varepsilon>0: \forall 0 \leqq x<\varepsilon: \mathscr{D}(x)=0 .
$$

Up to now, this has only been proven for sufficiently high temperature $[14,15]$ and for some particular models (e.g. [16]). This means that, at least for high enough temperature, one has to select among the class $L$ of characteristic functions those which are analytic. This constitutes already a rather strong restriction.

Other results of this type would be interesting to understand which subclass of the class $L$ is to be connected with $d$-dimensional Ising ferromagnets.

\section{Conclusions}

The probabilistic approach of the $d$-dimensional ferromagnets has always been considered as a problem of dependent random variables. In this paper, we have proven that, at the thermodynamic limit, the free-energy density $g(\beta, t)$ of such models with pairwise interactions and for a large class of free spin measures, is such that the function $\phi(t)=\exp \{\beta g(\beta, t)-\beta g(\beta, 0)\}$ is the limit characteristic function of a suitably renormalised sum of independent and non-identically distributed random 
variables, whatever the temperature $\beta^{-1}$ (except possibly for the Cauchy case).

In particular, we have established additional restrictions for our characteristic functions $\phi(t)$ within the class of $d$-dimensional Ising ferromagnets.

We have also shown how non-trivial inequalities between the Ursell functions above the critical temperature (if it exists) are direct consequences of the canonical representation formulae of such a characteristic function. This method also leads to new inequalities.

So far, our proofs rest essentially on the Lee-Yang theorem but they may well turn out to have a more general character. This aspect of the problem is currently under investigation.

Acknowledgements. The author is grateful to D. B. Abraham, J. Bricmont, Ph. de Gottal, F. Dunlop and C. Itzykson for interesting discussions.

\section{References}

1. Yang, C. N., Lee, T. D.: Statistical theory of equations of state and phase transitions. I. Theory of condensation. Phys. Rev. 87, 404-409 (1952)

2. Lee, T. D., Yang, C. N.: Statistical theory of equations of state and phase transitions. II. Lattice gas and Ising model, Phys. Rev. 87, 410-419 (1952)

3. Baker, G. A. Jr.: Some rigorous inequalities satisfied by the ferromagnetic Ising model in a magnetic field Phys. Rev. Lett. 20, 990-992 (1968)

4. Newman, C. M.: Inequalities for Ising models and field theories which obey the Lee-Yang theorem. Commun. Math. Phys. 41, 1-9 (1975)

5. Lieb, E. H., Sokal, A. D.: A general Lee-Yang theorem for one-component and multicomponent ferromagnets. Commun. Math. Phys. 80, 153-179 (1981)

6. De Coninck, J., de Gottal, P.: New inequalities for Ising ferromagnets. J. Stat. Phys. (to appear)

7. Griffiths, R. B.: In: Phase transitions and critical phenomena. Domb, C., Green, M. S. (eds.), Vol. 1. London, New York: Academic Press 1972

8. Petrov, V. V.: Sums of independent random variables Berlin, Heidelberg, New York: Springer Verlag, 1975

9. Gnedenko, B. V., Kolmogorov, A. N.: Limit distributions for sums of independent random variables. Reading, Mass.: Addison-Wesley 1954

10. Sokal, A. D.: More inequalities for critical exponents. J. Stat. Phys. 25, 25-50 (1980)

11. Gnedenko, B. V.: The theory of probability. Translated from the Russian by Seckler, B. D. New York: Chelsea Publishing Company 1968

12. Mitrinovic, D. S.: Analytic inequalities. Berlin, Heidelberg, New York: Springer-Verlag 1970

13. Whittaker, E. T., Watson, G. N.: A course of modern analysis. Cambridge: Cambridge University Press, 1965

14. Gallavotti, G., Miracle-Sole, S., Robinson, D. W.: Analyticity properties of a lattice gas. Phys. Lett. 25A, 493-494 (1967)

15. Bessis, J. D., Drouffe, J. M., Moussa, P.: Positivity constraints for the Ising ferromagnetic model. J. Phys. A 9, 2105-2124 (1976)

16. Abraham, D. B., De Coninck, J.: Yang-Lee zeros of a planar Ising model (in preparation)

17. Fisher, M. E.: Rigorous inequalities for critical-point correlation exponents. Phys. Rev. 180, 594-600 (1969)

18. Titchmarsh, E. C.: The theory of functions. Oxford: Oxford University Press, 1979

19. Breiman, L.: Probability. Reading, MA: Addison-Wesley Publ. Comp. 1968

Communicated by J. Fröhlich

Received March 3, 1984 
